\title{
Prototypical Reasoning about Species and the Species Problem
}

\author{
Yuichi Amitani
}

\begin{abstract}
The species problem is often described as the abundance of conflicting definitions of species, such as the biological species concept and phylogenetic species concepts. But biologists understand the notion of species in a non-definitional as well as a definitional way. In this article I argue that when they understand species without a definition in their mind, their understanding is often mediated by the notion of good species, or prototypical species, as the idea of "prototype" is explicated in cognitive psychology. This distinction helps us make sense of several puzzling phenomena regarding biologists' dealing with species, such as the fact that in everyday research biologists often behave as if the species problem is solved, while they should be fully aware that it is not. I also briefly discuss implications of this finding, including that some extant attempts to answer what the nature of species is have an inadequate assumption about how the notion of species is represented in biologists' minds.
\end{abstract}

Keywords Definition · Good species · Prototype theory $\cdot$ Species problem

\section{Introduction}

A book on the history of the so-called "species problem" would probably begin with the advent of the new

Note: This is the penultimate version of the published paper (Amitani, Y., 2015, Prototypical Reasoning about Species and the Species Problem, Biological Theory). But since in the published version many paragraphs are unintentionally divided to a miscommunication in the editing process, you may find this version more readable.

Tokyo University of Agriculture

Abashiri, Hokkaido, Japan

E-mail: yuiami@gmail.com systematics and the biological species concept (BSC), and continue with the many other definitions that followed in its wake. Wilkins (2009a) cites more than 20 definitions of species in his book on the history of the concept of species.

But this perspective on the history of the species problem would only illuminate one aspect of it, because biologists often deploy the notion of species without advocating a particular species definition. This fact about biologists' use of species was observed by naturalists as early as Charles Darwin (1859, p. 44).

No one definition [of 'species'] has as yet satisfied all naturalists; yet every naturalist knows vaguely what he means when he speaks of a species. (italics added)

Darwin's remark suggests there are two different modes of understanding the concept of species:

1. Biologists may understand species through definitions 11

2. Biologists may understand species through non-definitional means (Darwin calls this a "vague" way of understanding).

Biologists have done both.

This article aims to show that Darwin was by and large right regarding biologists' thinking about species. This will involve turning to the psychological literature concerning prototype thinking, and how this sheds new light on the species problem. In particular, I suggest that the concept of good species, a prototype of species, mediates the non-definitional, or perhaps implicit mode of understanding of species. A good species is a taxon judged to be a species according to more than one species criterion_ such as reproductive isolation

1 Names of concepts are written in italics. 
and phylogenetic properties_— or a taxon judged generally to be a species by competent biologists, whether the phrase merely expresses one's epistemic confidence in the taxonomic judgment or has even an ontological implication that the species category is divided into good and not-so-good species. I will explain how these uses of "good species" involve reasoning about species in non-definitional ways.

This is not just describing one aspect of biologists' reasoning about species. Instead, it helps illuminate why biologists cannot reach a resolution of the species problem and what we should do to do so. For one, I enlist this idea to argue that many extant accounts of the species problem pay excessive attention to the incompatibility of individual species definitions, and overlook the notion of good species. Several authors, for example, have proposed radical solutions to the species problem such as that "species" does not constitute a single coherent concept_— based on the assumption that individual definitions of species are incompatible. However, biologists recognize that good species and the general concept of species play significant epistemological roles in their research.

The structure of this paper is as follows. In the next section, I briefly review a psychological concept of prototype as explicated in cognitive psychology. In the third section, I characterize the notion of good species in more detail. I suggest that good species is a prototype of the concept species and that biologists occasionally represent the concept of species by good species. This is an example of what Kahneman and Frederick (2002) call attribute substitution. In the fourth section, we will see how attribute substitution can make sense of the way in which biologists work with the concept of species and the species problem. Finally, I briefly discuss how this finding presents problems with several extant attempts to describe and solve the species problem.

\section{Prototype as a Psychological Concept}

Here I give a brief review of prototype theory of concept and the concept of prototype as explicated in cognitive psychology. For a detailed overview of the psychological literature, see for example, Laurence and Margolis (1999) and Murphy (2002). I will also enlist several features of a prototype when I argue that good species is a prototype of species $(\$ 3.2$.

Cognitive psychologists began to pay serious attention to prototypes when they discovered prototype effects (Rosch and Mervis, 1975; Rosch, 1978). Prototype effects have been seen as a critical blow to the traditional theory of concepts, aptly dubbed the classical theory. According to the classical theory, a concept is represented by necessary and sufficient conditions for its application: a bachelor is an unmarried man. One implication of this theory is that every instance of a concept is treated the same way in our mind as long as it satisfies those conditions: if Tom and George are both bachelors, then our mind represents them in the same way in this regard. Prototype effects provide strong evidence against this picture. "Clear" or prototypical instances of a concept and "unclear" or atypical ones may be represented differently in our mind. When subjects undertake various tasks involving concepts (e.g., naming instances of a concept or judging the membership of a particular instance of a concept), their responses often differ depending on whether or not they are dealing with prototypes of a category. There are a number of examples of this: subjects require less time to identify a typical member of a category (e.g., a dog for pet), than an atypical member (e.g., a snake); young children learn category membership of typical members first (i.e., they tend to learn that apple is a fruit before learning that so is olive); when subjects are asked to list members of a category, they tend to name typical members first, and more frequently.

From these results, researchers like Eleanor Rosch put forward the prototype theory of a concept: a concept is associated with a cluster of features and each feature is weighted by the frequency with which it is exemplified by the members of the category. For example, fruit is associated with features such as being sweet and brightly colored, and those features are exemplified with different frequencies by different kinds of fruits. A prototype is a highly exemplary instance of a concept by virtue of possessing a sufficient number of those features, as apples are prototypical fruits because they have so many of those properties. This explains the graded membership of instances of a concept, because different members of the category fruit have a different number of those features. The prototype theory also explains how subjects react to different kinds of fruit differently. It takes less time to categorize apples as a fruit than olives because subjects can quickly and confidently recognize that apples are more similar to other members of fruits than those of vegetables, since apples have many important features of fruit. Although there are several objections to the prototype theory (see Laurence and Margolis, 1999, for the objections), it is one of the leading accounts on concepts in cognitive psychology ${ }^{2}$

${ }^{2}$ See Footnote 10 on whether the objections affect the arguments in the present article. 


\section{Notion of 'Good Species'}

In this section I apply the psychological notion of prototype to the concept of "good species." "Good species" is a rather unofficial technical term used in systematics and contemporary biology in general. One often sees it used in taxonomic description papers and scientific papers on topics related to species, such as speciation. "Good species" is typically used to characterize individual species, as in "Xus bus is a good species." Once a taxon is considered to be a good species, the fact that it is a species is generally taken for granted. This is partly because being deemed a good species implies that it will satisfy conditions specified by many species criteria and thus be judged to be a species under them: Xus bus is a reproductively isolated population, consists of a monophyletic lineage, occupies a single distinctive niche, and so on at the same time. Thus Xus bus will be judged to be a species under the biological species concept, the ecological species concept, the monophyletic species concept, and so on so that it looks as though it would be a species regardless of one's theoretical commitments 3

\subsection{Meanings of "Good Species"}

Here I describe a couple of usages of "good species" among biologists. Among the several usages of "good species," our focus is on a case in which it is used to refer to (1) a species which is distinctive or well-defined by multiple species criteria, or (2) a taxon that an author assumes is generally classified as a species by the biological community.

An Alleged Species by Multiple Criteria A taxonomist sometimes implies that multiple alternative species criteria are satisfied by a good species. The papers in which this usage occurs tend to make the fact that their judgment is based on multiple criteria explicit. One example of this is as follows (Hamada and Adler, 1999, p. 273):

Polytene chromosomes of four members of the Simulium perflavum species group in Brazil are described,... Chromosomal, morphological and ecological evidence indicates that $S$. maroniense Floch

\footnotetext{
3 Note that "good species" is an unofficial term and thus its usages may greatly vary from one biologist to another. Thus I do not claim that this is the only usage of the term. What I will do below is to suggest that biologists do use "good species" in a certain way, but this doesn't preclude the possibility that biologists use the term in other ways. See Footnote 6 for such a usage.
}

\& Abonnenc, previously considered synonymous with $S$. rorotaense, is a good species.

In this case the authors cite three alternative species criteria (genetics, morphology, and ecology) when they call the taxon a "good species."

This might look like definitional reasoning, but none of the extant, competing definitions of 'species' is invoked as the correct definition. Nor is there any claim that the range of criteria satisfied constitute necessary and sufficient conditions for specieshood. It is rather the case that the more criteria satisfied, the more confident biologists are in their taxonomic judgment.

Some species theorists seem to follow this usage of "good species," too. Alan Templeton (1989) proposes his own definition of species, the cohesion species concept. The rough idea is that a species is a population which is reproductively isolated from others and/or occupies a distinct fundamental niche (and thus is subject to the common selection pressure). He argues that extending the biological species concept this way we can include asexual organisms and thereby have a more comprehensive picture of evolutionary processes behind species and speciation. What is important in our discussion is that Templeton regards it as an advantage of the concept that it helps us make sense of why a good species is good. For Templeton, good species "are generally regarded as geographically cohesive taxa that can coexist for long periods of time without any breakdown in genetic integrity" (1989, p. 23). Then he argues that in order to live together for a sufficiently long time in a single habitat, those taxa need to be reproductively isolated and occupy different niches.

The fact that there is no breakdown in genetic integrity in spite of sympatry implies the lack of genetic exchangeability [i.e., reproductive isolation] between taxa. However, the condition of prolonged coexistence also implies that they have distinct ecological niches. Hence, "good species" are those that are well defined both by genetic and demographic exchangeability. (1989, p. 23; italics added)

Templeton then goes on to compare good species with "bad" species, those which satisfy either reproductive or ecological criteria, but not both. "By providing a precise definition of 'bad species' (the conflict between genetic and demographic exchangeability), the cohesion concept is a tool for gaining insight into the process of speciation" (1989, p. 23). One can see that in his conception of "good species" the more criteria satisfied, the better a taxon counts as a species, as far as those reproductive and ecological criteria are concerned. 
Notice that Hamada and Adler and Templeton could mean slightly different things by "good species." This is because biologists may mean two different things by "good species." Firstly, they may use the phrase only to express epistemic confidence in their taxonomic judgment: when they call Xus bus a good species, they could mean that there are good grounds for their taxonomic judgment that it is a species (as opposed to a subspecies, a variety, and so on). This is only concerned with their epistemic state, not what is the case about a particular taxon. However, taxonomists could also mean that good and bad species are different on ontological grounds: they are different kinds of species. In the two quotes discussed, Hamada and Adler do not mention (at least explicitly) ontological differences between good and bad species, while Templeton does ${ }^{4}$

That biologists including Templeton regard it as a characteristic of good species that they satisfy multiple species criteria is interesting in light of work in cognitive psychology. Cognitive psychologists point out that humans are particularly keen to make multiple interrelational associations among objects. In other words, humans tend more quickly to learn how to tell one category from another when two categories are different in multiple properties, rather than a single property (Rosch and Mervis, 1975: Murphy, 2002 Holland et al, 1989). If one species is different from another in multiple criteria, then their phenotypic difference is so striking that one could easily classify them as different species.

Good Species as a Taxon Generally Judged to Be a Species by the Biological Community Another, related usage is the one in which it is used to refer to a taxon that an author assumes is generally classified as a species by the biological community. One difference from the above usage is that when a biologist uses "good species" this way, she does not mention any particular criterion of species classification, but simply takes it for granted that the biological community would agree that a taxon at hand is a species. While the biologist might believe that this classificatory judgment is based on some criteria, she rather emphasizes collective agreement. To put it another way, this usage is based on the second-order judgment, one's judgment about other taxonomists' judgment.

While taxonomists would and should rely on their own judgment, not what other taxonomists have said, when they describe a new species, they do make reference to collective judgment in some contexts. For instance, under this usage, biologists often use a good

${ }^{4}$ I thank an anonymous referee for bringing my attention to this point. species as a a source of further induction. That is, the authors assume that "good species" are species and try to discover novel characters of them in order to infer something significant about the nature of species in general. Kai Chan and Simon Levin seem to use "good species" in this way. In so doing, they attribute some properties (exchanging genetic material with each other) to species in general.

It is commonly assumed that "good" species are sufficiently isolated genetically that gene genealogies represent accurate phylogenies. However, it is increasingly clear that good species may continue to exchange genetic material through hybridization (introgression)... (Chan and Levin 2005. p. 720)

In cases like this, the authors do not always cite any paper to support that the relevant "good species" are, indeed, actually recognized as species; it is implied that they are so recognized by the taxonomic and biological community, and fellow biologists would not find it difficult to see that implication.

The above authors do not explicitly discuss species definitions. But James Mallet (1995a b, 1996) uses "good species" in a similar way, while discussing species concepts.

I used the term 'good' species several times meaning that people generally agree that 'the blue whale' and 'the fin whale,' for example, are species, ... Unless taxonomists are mad, there is something reasonable about such species ... (Mallet, 1996, p. 174)

He does not refer to any particular criterion when he says that the blue whale and the fin whale are recognized as a species. Mallet uses this phrase independently of any particular criterion, because he intends to offer his definition of species (genotypic cluster species concept) by examining what features good species possess. Note that Chan \& Levin do not go that far; they do not rule out the possibility that biologists adopt different definitions of species when they classify some taxon as a good species ${ }^{5}$

\footnotetext{
5 I am inclined to offer an interesting instance of this usage of "good species." Arthur Cronquist (1978), a plant taxonomist, quotes, as "an old joke" among taxonomists, "a good species is what a good taxonomist says it is" and compares this with a statement on pornography made by Potter Stewart, a judge of the Supreme Court of the Unites States, that "hard-core pornography" was hard to define, but "I know it when I see it." The same comparison is made by Pigliucci and Kaplan (2006) (without mentioning Cronquist), although they simply use the term "species," not "good species." Here Cronquist and Stewart both appeal to collective coincidence
} 
It is also worth noting that there is a normative element to the notion of good species. Characterizing a taxon by calling it a "good species" often involves a (perhaps reasonable) expectation that other taxonomists will or should recognize the taxon as a species. Mallet emphasizes how normativity arises from collective agreement within the taxonomic community. When he says, "Unless taxonomists are mad, there is something reasonable about such species," Mallet implies that there is a normative element to the collective judgments of taxonomists.

Summary We have seen two important usages of "good species" among biologists. Among other things, the phrase "good species" refers to:

1. an alleged species that satisfies more than one species criterion, and

2. a taxon generally recognized as a species by the biological community.

Although the two meanings are different, they overlap significantly, in that if a taxon is a good species in the first sense, it is likely to be a good species in the second sense, and vice versa. The more species criteria - such as reproductive isolation and phylogenetic properties - a taxon satisfies, the stronger the expectation that other taxonomists would also classify it as a legitimate species will be, whether the expectation is based on ontological grounds or not. The reverse relation also holds: if Xus bus is generally judged to be a species by the taxonomic community, then Xus bus is judged to be a species according to many criteria. ${ }^{6}$

\subsection{Good Species is a Prototype of Species}

Now I propose to analyze this notion of good species, drawing on some tools from cognitive psychology. My thesis is that the notion of good species is a prototype

of judgments on membership of the categories_-if Judge Stewart had not believed that other people would agree with his judgment, one might ask why he made such an argument because the appeal to his intuitive judgment would not be persuasive at all.

${ }^{6}$ It is important to remember that I do not intend to say that this is the only way in which biologists use the phrase, and that some of the other usages in fact do not fit with the one discussed here. For example, biologists often do use "good species" to refer to a taxon if it satisfies a single species criterion rather technically (see Dumont (2004) for one instance). It should come as no surprise given that "good species" has no official definition. The point is, however, that biologists often do use the phrase in the way I have suggested, and this usage can be subject to analysis in terms of psychological theories of concepts, as I will do in the next section. of species, as explicated in cognitive psychology, and biologists often represent species with its prototype, good species, when they think of species non-definitionally. I will briefly review some of the prototype effects and demonstrate that the notion of good species shares those features. Due to the form of my argument, each point of similarity, if taken alone, may not be strong enough to make a convincing case for my thesis; however, those points, if brought together, strongly suggest that good species is a prototype of "species."

'Good $X$ ' The term 'good species' has the same linguistic features as other prototype terms. Quite literally, the phrase "good $X$ " is often used by psychologists to refer to prototypical instances of a concept. When psychologists attempt to find prototypes of a concept operationally, they almost always ask subjects to pick "good" instances of a concept.

For example, (Armstrong et al, 1983, p. 277) use "good $X$ " to refer to a prototypical member of a category in the instructions of their experiment:

On this form you are asked to judge how good an example of a category various instances of the category are ...

There are many other examples like this. In an experiment by Smith et al (1988), subjects are asked to rate each item "for how good an example it is of the category" (p. 502). Psychologists themselves also commonly refer to a prototype by 'good $X$.' For example,

"subjects overwhelmingly agree in their judgments of how good an example or clear a case members are of a category ..." (Rosch, 1978, p. 36 , see also p. 37 )

"... The top half of the table contains the data for instances that were 'good' members of their corresponding conjunctions ..." (Smith et al 1988 , p. 504).

Notice that Smith et al add quotation marks to "good members." The use of quotation marks even coincides with taxonomists' use of them. Many biologists add quotation marks to "good" or "good species," as Mallet and Chan and Levin do. The use of scare quotes generally indicates that the speaker distances himself from the literal meaning of a word or phrase enclosed within them. Thus when biologists use scare quotes to "good species," they try to distance themselves from the literal meaning of the phrase. Still, it is not easy to say what exactly biologists distance themselves from and why when sufficient contextual information is not available. This is because what scare quotes contribute 
to the informational content of an entire utterance depends on the context in which it is made (see Predelli, 2003).

Nevertheless we can put forward a more or less plausible account of the coincidence. First I want to look at parallelism with another usage of scare quotes with regards to prototypes. Psychologists do not use scare quotes only for "good X"; they also use them for adjectives derived from a category name such as "doggy." For example, Armstrong et al (1983, p. 275) use such an adjective in the instruction to subjects (they took this part of the instruction verbatim from Rosch 1975):

This study has to do with what we have in mind when we use words which refer to categories .... Think of dogs. You all have some notion of what a 'real dog', a 'doggy dog' is. To me a retriever or a German Shepherd is a very doggy dog while a Pekinese is a less doggy dog. ... On this form you are asked to judge how good an example of a category various instances of the category are ..

Using scare quotes, psychologists distance themselves from the literal meaning of "a doggy dog" because it sounds self-contradictory; after all, dogs are dogs, and they are all doggy by definition. By the same token, they also distance themselves from the literal meaning of "real dog" or "good dog," because no dog is, by definition, more or less real (or good) as a dog than others. It appears that behind intuitions like those is the classical view of concepts. In other words, what the scare quotes imply is that psychologists are more or less pluralistic on concepts when they use scare quotes — the classical view and the prototype view_ and this is why they use scare quotes for "a doggy dog" and "good X."

I suspect that this same is true of biologists using scare quotes to "good species." As the quote from Darwin at the outset of the paper suggests, biologists may be pluralistic on the notion of species. They often have in mind prototypes when they talk about species (the prototype view). Meanwhile, biologists do represent the notion of species via definitions (the classical view). Thus it is no wonder that they distance themselves from the literal meaning of "good species" while using prototype reasoning. If this account is on the right track, then the use of scare quotes by psychologists and biologists may not be just a coincidence; they use scare quotes for the same reason.

Hedges More linguistic evidence comes from hedges. Although "A robin [a prototypical bird] is a bird" and "A penguin is a bird" are both true, adding some hedges (qualifying terms such as "virtually," and "technically") could change their truth values: "A penguin is technically a bird" is judged to be true while "A robin is technically a bird" is not (Lakoff, 1973, Rosch, 1978). To put it differently, a prototype will never be a borderline case. The same thing seems true of "good species." Recall that Templeton distinguishes good and bad species. Ernst Mayr joins him when he distinguishes good species and borderline cases (incipient species) in his 1942 book (Mayr, 1942, p. 155):

Geographic speciation is thinkable only, if subspecies are incipient species. This, of course, does not mean that every subspecies will eventually develop into a good species. Far from it! All this statement implies is that every species that developed through geographic speciation had to pass through the subspecies stage. There is, naturally, a considerable infant mortality among subspecies and only a limited number reaches adulthood, or the full species stage 7

Thus, for Templeton and Mayr, a good species is not a borderline case of species (see also Coyne and Orr (2004) for another instance). Consequently, those hedges would change the truth value of propositions containing "good species." Sentences such as "Xus bus is a good species and technically a species" sound false in the usage discussed here. In contrast, "Xus bus is not a good species, but technically a species" sounds true, just as "A penguin is technically a bird" sounds true. I concede that there is a usage of "good species" in which hedges do not change the truth value of propositions containing the phrase. Nonetheless there is also a usage of the phrase in which hedges do change the truth value of some propositions containing 'good species.'

Agreement on Specieshood Related but nonlinguistic support for our claim is agreement among biologists on specieshood. Rosch (1978) points out that subjects make similar judgments on how well a given object belongs to a category. This implies that a prototype of a category is generally considered to be a member of that category. This is what is observed about good species. We have seen that once a biologist judges some taxon to be a good species she will form a strong expectation that other competent biologists will concur. Biologists often use "good species" to refer to species taxa that almost all biologists would judge to be a species, as Mallet does.

7 One might object that Mayr may in fact see incipient species as non-species and thus think that there are no borderline cases. But this is not the case. See Mayr 1942, p. 114 
Inference from Good Species Biologists sometimes infer properties of a good species to the whole group of species, including borderline "bad" species. For example, Chan and Levin (2005) point out that some good species hybridize with each other and then suggest that species in general could hybridize (see the quote from them on p. 4) ${ }^{8}$ Psychologists found that subjects tend to infer properties exhibited in a prototypic member of a category to non-typical members (but not the other way around): if a robin has a property $f$, then a penguin probably has it (but not vice versa; Lakoff, 1989). It is worth noting that Lakoff's remark also shows that this inference would be made for all members of the category, not just atypical members, because a category generally consists of "good" (prototypic) and "bad" (non-prototypical) members. Subjects tend to believe that if a robin has a property $f$, then any bird (including a "bad" member of bird like penguin) would have it. If it is sensible to read Lakoff's remark this way, people arguably make similar inferences involving good species. In other words, biologists would infer the properties a species has from those a good species has, which is what we have observed 9

One last point. In the preceding section (\$3.1), we have observed that the notion of good species has epistemic and ontological dimensions. Taxonomists may call a taxon a "good species" only to express their epistemic confidence on its specieshood; whereas they could also imply that there are real or ontological differences between good and bad species as Templeton does. It is important to note that psychologists' conception of prototypes has both of the elements. We have seen that prototype theorists assume that a category has the ontologically graded structure $(\$ 2$. On the other hand, several prototype effects mentioned before, such as quick identification of prototypical members of a category, imply that one has epistemic confidence or clarity about

8 The use of prototypes in taxonomic practice may be more widespread than this section suggests. Jody Hey (2001, p. 162) points out that when taxonomists engage in species classification, they often rely on a prototype of that species and treat each species as if it is a natural kind, rather than an individual.

9 As I noted in this section, the prototype of species, good species, is characterized by the consilience of different properties. This makes our proposal look like one of the cluster views of species, including the homeostatic property cluster theory (Boyd, 1999) and David Hull's cluster definition of species (Hull 1965). The difference, however, lies in the fact that our proposal is not about the species category per se, but about its prototype. It can be the case that a category has a prototypical member while it has sharp boundaries: even number is a sharply delineated category but does have a prototypical member, such as 2 . Likewise our proposal does not preclude the possibility that the species category is not characterized by a cluster of properties. the category membership of such instances. Franks and Bransford (1971) also found that subjects are more confident in identifying prototypical items than atypical items. This is also reflected in the fact that many psychologists describe prototypical members as a "clear" example of a category. Eleanor Rosch (1978), for example, says, "Another way to achieve separateness and clarity of actually continuous categories is by conceiving of each category in terms of its clear cases rather than its boundaries" (pp. 35-36.). Psychologists' stress on diagnostic clarity of prototypical members implies that subjects have epistemic clarity or confidence in their judgment when they classify a prototypical member of a category.

\subsection{How Biologists Reason With the Help of Good Species_-Attribute Substitution}

Another interesting connection between good species and prototype reasoning has to do with the phenomenon of "attribute substitution" Kahneman and Frederick, 2002). When attribute substitution occurs, a subject represents one attribute of an object with another somewhat relevant, not identical, attribute. Kahneman and Frederick call the first kind of attribute the "target attribute" and the second the "heuristic attribute." In their words, "attribute substitution" means that "an individual assesses a specified target attribute of a judgment object by substituting another property of that object - the heuristic attribute — which comes more readily to mind" (p. 53, italics in original). Kahneman and Frederick suggest that this is often done by substituting questions; subjects often replace a question about a target attribute (a target question) with a question about a heuristic attribute (a heuristic question). If attribute substitution (along with substitution of questions) is one of the things subjects do in the case of prototype reasoning, and the good species category is a prototype of the notion of species, then it is no wonder that biologists do the same thing with good species. This is to say that biologists represent the notion of species by its prototype, good species, in their minds and make an inference about the attributes a species could have (target attributes) from the attributes a good species has (heuristic attributes).

Attribute Substitution of Species by Good Species: Biologists often implicitly represent the concept of species by its prototype, good species, and infer what attributes a species has (target attributes) from the attributes a good species has (heuristic attributes). Thus, biologists often implicitly answer a question about a species (a target question) by answering a question about a good 
species (a heuristic question).

This is because attributes of a good species are often more readily available in the biologist's mind and this leads him to access those attributes when he reasons about the species category.

Of course, what we have done here is just to follow Kahneman and Frederick and postulate the existence of the substitution of species with good species. In the next section I will make a case for it by pointing out that attribute substitution can make sense of a couple of puzzling phenomena regarding biologists' dealings with species and the species problem 10

\section{Non-definitional Understanding of Species}

In this section we will see how good species as a prototype of species and attribute substitution could explain some puzzling phenomena regarding biologists' dealings with species in a non-definitional mode.

One feature of the non-definitional mode of understanding species is what might be called elusive transparency. Several researchers have observed that biologists believe they understand the nature of species when they think about it in an unarticulated way, but subsequently find themselves at a loss when asked to define it: in everyday research biologists behave as if the species problem is already solved, while fully aware that it isn't. Although this kind of phenomenon might be seen in other areas, philosophers have not paid due attention to it in the controversy over the concept of species.

\subsection{Hey's Observation}

This phenomenon has been observed by biologists themselves on several occasions. Geneticist Jody Hey pro-

10 In this section I applied the prototype theory of concept to analyse biologists' attitudes toward the concept of species. One might suspect that this was only possible if I downplay or ignore the fact that there are several objections to the prototype theory in cognitive psychology (see, for example Laurence and Margolis, 1999, for the objections). Yet this does not mean that the use of the prototype theory in the present paper is not warranted. For our purposes, it is not necessary to assume that the prototype theory is the appropriate account of concept. Our proposal is compatible with pluralism about the concept of concept. It is because our aim is to point to some unattended aspects in biologists' use of the concept 'species' and to explore their philosophical implications. In this context, we need an assumption that the prototype theory accounts for the phenomena in question, but not an assumption that it accounts for all the phenomena about concept. And pluralism about concept is a sensible position. We cannot go into the details of the debate, but several authors are sympathetic to the pluralistic account of concept (see for example Machery, 2008). vides one example. Hey (2001) reports that biologists, including himself, often find themselves casually using the word "species" in conversation with colleagues, as if they fully understand its common meaning. And this despite the fact that they know, as intimately as anyone, all the difficulties that have attended every attempt to define the notion. In his own words, Hey confesses,

It has been my experience —and I am guessing that it is a typical one that when talking with biologists, one hears [the term "species"] tossed about regularly in a manner that supposes there is one single common meaning. If pressed on that common meaning, biologists are stuck, but they persist in using the word in a casual way much as laypersons do, as if it has a well-known meaning. (Hey, 2001, p.11)

This strikes much the same chord as Darwin's passage, written nearly 150 years earlier (\$1). Biologists still claim to know what "species" means, and take this understanding for granted in casual conversation. And, equally, biologists still find it hard to articulate their understanding to others, much less to give the single correct definition of the term. This is a rather puzzling phenomenon; one may wonder why biologists believe that "species" has one common meaning when they should be aware of the fact that there have been different attempts to define "species" in the history of biology and none commands universal support 11

Note that elusive transparency should not be taken to imply that everything biologists believe they understand about species is illusory. Biologists — from those mentioned in Darwin's quote to those working at present_ are not ignorant of the nature of species, not completely, anyway. Thus, biologists do know something about species. It simply doesn't take the form of a definition.

\subsection{Luckow's Observation}

A similar observation can be made specifically of taxonomists. Rarely do they specify which species concept they are using in papers describing new species. But of course, they, of anyone, can appreciate the variety of species concepts.

This is Melissa Luckow's observation. Luckow (1995) surveyed 130 papers on species in two professional journals for systematists, Systematic Botany and System-

11 Cronquist (1978) and Pigliucci and Kaplan (2006) also point to this apparent transparency of the notion of species to biologists. 
atic Zoology (now called Systematic Biology) from 19891993. In doing so, she observed that the authors of many papers do not specify what species concept they are using, despite an apparent expectation that they do so. Luckow says,

... most of the papers were not explicit about which species concept was being used. (p. 598) Only $20 \%$ of the papers in Systematic Botany were explicit as to the criteria being used; the default criterion was usually gaps in qualitative variation. An even smaller subset (8\%) of papers specified which species concept was being used. (p. 600)

Based on her study, Luckow recommends that systematists explicitly declare their choice of criteria for species classification. But it is puzzling why Luckow has to make such a recommendation in the first place; since taxonomists should be aware that there are different definitions of "species," they may well clarify what they mean by "species" by declaring their choice of definition. This observation conforms with Hey's anecdotal report that biologists tend to take their notion of species for granted and not feel the need to make it explicit in practice at least not unless they are asked to do so.

One might wonder if it is because of lack of space, not lack of interest or attention, that taxonomists do not articulate their choice of species concept. This may be true of some papers, but certainly not most. Declaring one's choice would not take much space at all, if one does not discuss what is the right concept of species. A few sentences or a couple of paragraphs would suffice, and many papers describing new species are three to five pages long. Thus, adding a few sentences to indicate their preferred definition would not much reduce - if at all — the possibility of acceptance. Therefore, I do not believe that taxonomists fail to articulate their choice because they fear this will cost them acceptance of their paper.

4.3 The Idea of "Good Species" Makes Sense of the Non-definitional Mode of Understanding Species

Our explication of "good species" helps us to better understand this non-definitional mode of understanding species. First, substitution of the concept of species by its prototype makes sense of Jody Hey's observation: that when he and other biologists participate in casual conversation, they employ the term "species" as if it had one common meaning, although they are fully aware of the fact that there is no universally accepted definition of "species." This makes sense if they represent the concept of species by a prototype and answer a target question (e.g., "Is species a homogeneous category such that biologists can easily grasp the nature of it?") by answering a heuristic question (e.g., "Is good species a homogeneous category such that biologists can grasp the nature of it?"). This is because a good species usually looks quite homogeneous and distinct in that it tends to satisfy many species criteria and one can easily tell that it is a species. We have seen that humans tend more quickly to learn how to tell one category from another when two categories are different in multiple properties, rather than a single property (see p. 3. Even though there is no clear and common meaning in the term "species" (a target attribute), the prototype makes it look as if there is such a meaning (a heuristic attribute), and speakers easily believe that they grasp it. This also explains why they find it hard to precisely define species; when species is represented by the prototype, their understanding of the notion is not mediated by words and a prototype may not easily translate into a strict definition_ the category has the gradient of membership.

Another fact Kahneman and Frederick notice about attribute substitution supports our idea that biologists often represent the notion of species by its prototype. They note that one condition for the occurrence of attribute substitution is that substitution is not intervened by a reflective psychological process (Kahneman and Frederick, 2002, p. 54). Thus, one has good reason to suspect that biologists make the same kind of substitution when engaged in casual conversation (recall Hey's observation quoted on p. 8), because they are probably not attending to details about species. This also suggests that good species is a component of the implicit mode of understanding species.

Attribute substitution also makes sense of observations made by Luckow. She notes that taxonomists are not interested in explicitly declaring their preferred species concept in their description papers. If the substitution occurs unreflectively, then naturally they would feel little need to spell out their conception of species and get involved in the controversy, because substitution may automatically lead them to believe, by the help of the prototype, that they understand the nature of species. A category mediated by its prototype looks transparent to a subject (a heuristic attribute), even though the nature and the right definition of species has been the subject of a considerable controversy (a target attribute). One can also describe this in terms of species criteria. A good species is a species according to many criteria. If a taxonomist recognizes a given taxon as a good species, she may feel little need to specify species criterion she would apply, because that taxon will be judged to be a species anyway. 
If the substitution occurs, we can make sense of the observations made by Luckow. And there is a piece of evidence that it does occur. Taxonomic practice helps the substitution occur in the minds of taxonomists when they describe a new species as a species. There is a recommendation in the taxonomic community that a (new) species should be described clearly, so that it can be easily distinguished from other species (Winston, 1999). Described this way, individual species are likely to be represented as a good species in the minds of taxonomists, because distinctness is a feature of a good species (see section 3.1). Substitution also nicely meshes with Hey's remark that taxonomists use prototypes in describing individual species (see Footnote 8 for the quote from him). If taxonomists make use of prototypes in their minds when they classify organisms into individual species, then they may well use them at the category level. Notice I do not mean that attribute substitution is always the cause of the phenomena observed by Luckow; a biologist may not make explicit his choice of species definition because of a deliberate decision. Yet the attribute substitution is still a possible, but often neglected explanation of many such cases.

There is one further thing to note. I suspect that taxonomists often commit attribute substitution when, for instance, they describe a new species as a species. This is true of many taxonomists, but not all biologists, especially species theorists engaged in the species controversy (certainly, a biologist such as Ernst Mayr would be sensitive to species definitions in any sort of research). Recall that Luckow's observation is about taxonomists in general, not participants in the species controversy. But where average taxonomists are concerned, they undertake their research relatively unbothered by the species problem.

\section{5 "Good Species" and the Species Problem}

The conclusion of the last section is that biologists frequently represent the concept of species by good species. In this section, I discuss two implications of this finding.

Good Species and the Persistence of the Species Problem I will discuss how this nondefinitional mode of understanding species helps explain why the species problem persists.

Let me elaborate the question. Given that species is seen as an important concept in evolutionary biology and that biologists have reached no consensus regarding its nature and the right definition of it, one might suspect this lack of consensus presents serious obstacles to biologists. However, this does not seem to be the case. The study of speciation has made significant progress for decades (Coyne and Orr, 2004). Taxonomy as a whole does not seem to slow down because of the species problem. Indeed, as the observations by Hey and Luckow suggest, biologists behave as if they are not as much bothered by the current state of the species problem as one might expect, even though they should be aware of the problem. This contributes to the persistence of the species problem in the following sense: had biologists abandoned the concept of species altogether because of the bleak prospect of resolving the species problem, then the species problem would have ceased to exist. Since they do not stop using the concept of species, however, naturalists and philosophers are still motivated to decide what the nature and the right definition of species is 12

Attribute substitution provides biologists with a way to employ the notion of species but bracket the problems surrounding it. A good species, as a prototypical species, is expected to be judged as a species by most competent biologists or many species criteria. Thus, as long as they work on good species, those biologists would not face serious conceptual or practical difficulties surrounding the notion of species. For example, if a taxon Xus bus is a good species, taxonomists do not have to make explicit which concept they adopt when talking about the taxon, because it would be a species whichever definition is taken. This means that in many cases, biologists could take for granted that a taxon they are working on is a species under most definitions and thus would not be bothered by the species problem.

It also means that disputes over competing species definitions may not be as relevant to biologists' actual dealings with species as many species theorists believe. We cannot estimate what proportion of the entire collection of species are good species, but it may well be more than a small fraction of it. Then it follows that in many research contexts selecting one definition of species over another does not matter much to biologists' practice. While adopting one species definition over another would still affect how we draw boundaries between species in principle, it does not make a significant difference to the way in which biologists classify and study individual species in practice.

12 It should be noted that one might take the persistence question more narrowly. According to the present description, the resolution of the species problem is not restricted to agreeing on the nature of species. If, for example, the biological community comes to agree that the species category is not a natural kind and abandons the project of giving a definition of it altogether, this would also be taken as a "resolution" of the problem in this description, because the species problem would no longer appear as a "problem" to biologists. In other words, in our interpretation, one aspect of the persistence of the species problem is that biologists feel pressed to solve the problem in one way or another. 
There Is More to The Species Problem Than Definitions Let me turn to the second implication. Our analysis so far is mainly concerned with how biologists represent the concept of species. Biologists tend to understand the concept of species through its prototype and when they do, they often do not have particular definitions in mind. One might think that our enterprise is thus not directly concerned with the central questions of the species problem - what is the nature of species and what is the right definition of species_- and therefore that our analysis is not relevant to the species problem. But this is not entirely true; although the questions of how species is represented and what its nature is are not identical, they might not be independently answered. An answer to the latter question could restrict the range of possible answers to the former question.

Indeed, some extant attempts to answer the core questions of the species problem make an assumption about the way in which biologists represent species. Since the abundance of species definitions is a salient phenomenon in the history of the modern species debate, philosophers and biologists have paid excessive attention to individual definitions and their mutual incompatibility: the same taxon is a species according to one definition, but not a species according to another. Among those attempts, Marc Ereshefsky (1992) argues that the conflict among different definitions of species is so grave that we should only use individual definitions according to contexts - if we are interested in the phylogenetic relationship, we should use some version of the phylogenetic species concept (PSC), for example- and abandon the general concept of species (species eliminativism). There is a species according to BSC or PSC, but not a species per se ${ }^{13}$ Thomas Reydon (2005) goes so far as to conclude from the abundance and incompatibility of different definitions that "species" does not constitute even a single coherent concept; the term "species" is merely a homonym of different definitions.

What Ereshefsky and Reydon share is the assumption that if various definitions of "species" are inherently incompatible, then there could not or should not be a single concept of species. This assumption falls in line with the so-called classical view of concepts: a concept is represented and, more importantly, individuated by necessary and sufficient conditions for its application. Since different species definitions have different application conditions, they are different concepts and there is no common concept of species. What these

\footnotetext{
13 Recently Ereshefsky (2010) changed his position and now admits that the term "species" is so entrenched in biologists' practice that it is not practical to eliminate it. But he gives little analysis to the psychological and epistemological roles species plays in biologists' minds.
}

attempts overlook, however, is that biologists do not always mentally represent a particular definition when they talk about species. Instead, they are more likely to represent the concept of species with its prototype, i.e., good species (see a point Machery (2012) makes on the concept of life). If this is true, then it follows, for example, that it is too quick to conclude that "species" is a homonym just because different definitions are incompatible, because good species is a prototype of the general concept of species, not any particular definition of "species." Ereshefsky's eliminativism is also dubious, because it does not appreciate epistemological roles - such as a source of further induction — which the concept of good species plays in biologists' thinking (see also Ingo Brigandt (2003) and John Wilkins (2009b) for further discussions of the need to recognize a broader conception of species concepts).

\section{Conclusions}

The species problem revolves around competing definitions of species. However, in this paper, I have suggested that there is more to the species problem than competing definitions. Specifically, I have suggested that biologists understand the notion of species in a nondefinitional mode as well. Furthermore, I suggested that in the non-definitional mode, biologists understand species through the notion of "good species." The category good species is a prototype of species, as explicated in cognitive psychology. As in other cases of prototype reasoning, biologists engage in attribute substitution they infer what attributes a species has from the attributes a good species has. Thus, biologists often implicitly answer a question about a species by answering a question about a good species. And this makes sense of several puzzling phenomena regarding biologists' dealings with species. When biologists work on a good species, they do not get bothered by issues regarding definitions of species, because a good species meets various criteria of species at the same time and it is a species whichever definition one prefers. Finally, we have seen how the prototypical mode of understanding species illuminates problems with several current attempts to describe and solve the species problem.

A note is in order. It might be noticed that this study is not based on psychological experiments where biologists' actual reasoning about the material they are actually working on in their research is examined. It is partly because there is no experiment, as far as I know, on whether or how biologists hold the prototypical attitude toward the species category. This limitation is shared by many of the current studies on the cognitive basis of scientific reasoning (see, for example, Klahr 
2000 Nersessian, 2008) ${ }^{14}$ Even with this limitation, I believe that discussion in the present paper will shed a light on an unattended aspect of biologists' dealing with the concept of species and of the species problem.

Acknowledgements Versions of this paper were presented at several places including the University of Pittsburgh, the Kazimierz Naturalist Workshop (Kazimierz Dolny, Poland), the Annual Symposium of the Union of the Japanese Societies for Systematic Biology (National Museum of Nature and Science, Japan), the Japan-Korea Workshop on Philosophy of Science (Kyoto University, Japan), the Japan-Taiwan Philosophy of Biology Workshop (National Chung Cheng University, Taiwan), and the biannual meeting of the International Society for the History, Philosophy and Social Studies of Biology (Brisbane, Australia). I thank the participants for helpful comments. I am very grateful to the following people for improvements to this paper which I could not have made without them: John Beatty, Marc Ereshefsky, Chris Stephens, the late Brian Laetz, the research fellows of the Center for Philosophy of Science at the University of Pittsburgh, and two anonymous reviewers of this journal. This work is financially supported by JSPS KAKENHI (Grant Number: 25370016).

\section{References}

Armstrong S, Gleitman L, Gleitman H (1983) What some concepts might not be. Cognition 13:263-308

Boyd R (1999) Homeostasis, species, and higher taxa. In: Wilson R (ed) Species: new interdisciplinary essays. MIT Press, Cambridge, pp 141-185

Brigandt I (2003) Species pluralism does not imply species eliminativism. Philos Science 70:1305-1316

Chan K, Levin S (2005) Leaky prezygotic isolation and porous genomes: rapid introgression of maternally inherited DNA. Evolution 59:720-729

Coyne J, Orr H (2004) Speciation. Sinauer, Sunderland

Cronquist A (1978) Once again, what is a species? In: Biosystematics in agriculture, Rombergered Allanheld \& Osmun, Montclair, pp 3-20

Darwin C (1859) On the origin of species by means of natural selection. John Murray, London

Dumont H (2004) Distinguishing between the EastAsiatic representatives of paracercion weekers and dumont (Zygoptera: Coenagrionidae). Odonatologica 33:361-370

Ereshefsky M (1992) Eliminative pluralism. Philosophy of Science 59:671-90

Ereshefsky M (2010) Darwin's solution to the species problem. Synthese 175:405-425

Franks J, Bransford J (1971) Abstraction of visual patterns. J Experimental Psychology 90:65-74

14 They do conduct experiments, but subjects are typically non-scientists (in Klahr's experiments) and the experimental task is not about what scientists actually work on in their research (in Nersessian's case).
Hamada N, Adler P (1999) Cytotaxonomy of four species in the Simulium perflavum species group (Diptera: Simuliidae) from Brazilian Amazonia. Systematic Entomology 24:273-288

Hey J (2001) Genes, categories, and species. Oxford University Press, Oxford

Holland J, Holyoak K, Nisbett R (1989) Induction: processes of inference, learning, and discovery. MIT Press, Cambridge

Hull D (1965) The effect of essentialism on taxonomy - Two thousand years of stasis II. British Journal for Philosophy of Science 16:1-18

Kahneman D, Frederick S (2002) Representativeness revisited: attribute substitution in intuitive judgment. In: Gilovich T, Griffin D, Kahneman D (eds) Heuristics and biases, Cambridge University Press, Cambridge, pp 49-81

Klahr D (2000) Exploring science: the cognition and development of discovery processes. MIT Press, Cambridge

Lakoff G (1973) Hedges: A study in meaning criteria and the logic of fuzzy concepts. J Philosophical Logic $2: 458-508$

Lakoff G (1989) Cognitive models and prototype theory. In: Neisse U (ed) Concepts and conceptual development. Cambridge University Press, Cambridge, pp $63-100$

Laurence S, Margolis E (1999) Concepts and cognitive science. In: Margolis E, Laurence S (eds) Concepts: core readings. MIT Press, Cambridge, pp 3-81

Luckow M (1995) Species concepts: assumptions, methods, and applications. Systematic Botany 20:589-605

Machery E (2008) Doing without concepts. Oxford University Press, Oxford

Machery E (2012) Why I stopped worrying about the definition of life... and why you should as well. Synthese 185:145-164

Mallet J (1995a) Reply from Mallet. Trends in Ecology and Evolution 10:490-491

Mallet J (1995b) A species definition for the modern synthesis. Trends in Ecology and Evolution 10:294299

Mallet J (1996) Reply from Mallet. Trends in Ecology and Evolution 11:174-174

Mayr E (1942) Systematics and the Origin of Species from the viewpoint of a zoologist. Columbia University Press, New York

Murphy G (2002) The big book of concepts. MIT Press, Cambridge

Nersessian N (2008) Creating scientific concepts. MIT Press, Cambridge

Pigliucci M, Kaplan J (2006) Making sense of evolution. University of Chicago Press, Chicago 
Predelli S (2003) Scare quotes and their relation to other semantic issues. Linguistics and philosophy $26: 1-28$

Reydon T (2005) On the nature of the species problem and the four meanings of 'species.' Studies in History and Philosophy of Biological and Biomedical Sciences 36:135-158

Rosch E (1975) Cognitive representations of semantic categories. Journal of Experimental Psychology: General 104:192-233

Rosch E (1978) Principles of categorization. In: Rosch E, Lloyd B (eds) Cognition and categorization. Erlbaum, Hillsdale, pp 27-48

Rosch E, Mervis C (1975) Family resemblances: studies in the internal structure of categories. Cognitive Psychology 7:573-605

Smith E, Osherson D, Rips L, Keane M (1988) Combining prototypes: a selective modification model. Cognitive Science 12:485-527

Templeton A (1989) The meaning of species and speciation: a genetic perspective. In: Otte D, Endler J (eds) Speciation and its consequences, Sinauer, Sunderland

Wilkins J (2009a) Species: a history of the idea. University of California Press, Berkeley

Wilkins J (2009b) Species are not theoretical objects, or, on what there is in biology. Retrieved July 19, 2009 from http://philsci-archive.pitt.edu/ archive/00004835/

Winston J (1999) Describing species: practical taxonomic procedure for biologists. Columbia University Press, New York 